\title{
A Prototype Helping Device for the Visually Impaired Using an Optical to Mechanical Transducer
}

\author{
Garibaldi Pineda García \\ Facultad de Ingeniería Eléctrica \\ Universidad Michoacana de \\ San Nicolás de Hidalgo \\ Email: garibaldi@correo.fie.umich.mx
}

\author{
José Ortiz Bejar \\ División de Estudios de Posgrado \\ Facultad de Ingeniería Eléctrica \\ Universidad Michoacana de \\ San Nicolás de Hidalgo \\ Email: job@dep.fie.umich.mx
}

\author{
José Francisco Mercado Miramontes \\ Facultad de Ingeniería Eléctrica \\ Universidad Michoacana de \\ San Nicolás de Hidalgo \\ Email: ingjosefranciscomercado@ hotmail.com
}

\begin{abstract}
Computer hardware is becoming ubiquitous, from kitchen appliances to research machines to cell phones. This availability of computing capable circuits has driven its cost down, thus making several applications possible on commercial hardware. Computer vision is one of such tasks, it is now possible to create computer based helping devices for the visually impaired.

In recent years there has been an explotion of computer vision hardware for many applications, 3D scanners in particular which are used to interact with regular operative systems or games. This paper presents an apparatus which uses one of such devices (Kinect) developed by Microsoft that senses the world in 3D. This information is then processed with a low-power computer called the BeagleBone whose output will be sent to the user via mechanical pulses.
\end{abstract} paired.

Keywords-Kinect, BeagleBone, Computer Vision, Visually Im-

\section{INTRODUCTION}

Computer hardware is present in everyday tasks, from automated vacuum cleaners to washing machines to games. A recent increase in production facilities around the world has made it easy and affordable for independent groups to develop small computer systems. One of such computers is known as the BeagleBone, which was codeveloped by Texas Instruments workers and DigiKey. This small and power efficient computers are known as barebone computers [1].

Consumer electronics, particularly video game systems, have started using computer vision as a way to interact with the user. All three major game consoles (XBox 360, Playstation 3 and Wii) use some form of computer vision to change the more common button smashing interface for a user movement based one. The Kinect was developed to create a more natural user interface with the XBox 360 and has a significantly low price [2].

Given this conditions in the technological arena, one must wonder if people have done research to create a "visual prosthesis" with commercially available hardware. To our surprise, there's been very few work in this field of study [3].

In this paper an attempt to create one of such devices is presented. We intend to capture depth information via the Kinect sensor, then process it in the BeagleBone computer and give a vibratory output that the user can clearly identify. In section II we dive deeper into the characteristics of the components used to create our transducer. Section III will describe the planning and building phases. Some tests and their results are presented in section IV. Finally some conclusions and future research directions are given in section $\mathrm{V}$.

\section{COMPONENTS}

\section{A. BeagleBone}

The BeagleBone is one of the first of tinker-oriented barebones to hit the market. It was co-developed by Texas Instruments workers and the DigiKey company, this deal meant that DigiKey was to be the exclusive distributor at the beginning. Nowadays the BeagleBone can be purchased in several stores and it remains one of the most popular development environment boards. Its principal components are an ARM Cortex-A8 CPU running at 720MHz, $256 \mathrm{MB}$ of DDR-RAM, a wired ethernet port, a microSD slot, both host and client USB ports and multiple general purpose input/ouput (GPIO) pins. One key advantage of the BeagleBone is that it embraces the "physical computing" movement, partially started by the Arduino Community [4]. The fact that it has integrated GPIO, was the main reason to choose the BeagleBone for this project.

\section{B. Kinect}

The Kinect was developed as a more natural user interface for the XBox 360. It's main use is to translate users movements into actions inside a game environment or the console's operative system (OS). According to [2], it has a color RBG camera, an infrared projector and camera, plus four microphones. Further official information is to be purchased or reverse-engineered, the later was done by the OpenKinect Community [5]. They offered a bounty for the first hacker that could develop a working driver, this effort paid off and thus the Freenect driver was created. This driver works on all major OS and is being ported to the most popular hardware platforms as well. Since we used an ARM based platform and were restricted to the Linux OS, we opted to install an Ubuntu Linux version to be able to install the Freenect driver without hassle. The Kinect was designed for indoor use and it can estimate distances accurately from around 0.5 to 4 meters. 


\section{Micro-motors}

Our apparatus uses infrared light as its input (comming from the Kinect) and vibration pulses as outputs. We decided to use micro-motors with an unbalanced load, typically found in cellphones. The main reason to use them was that they use a small amount of power $(3.3 \mathrm{~V}$ and $100 \mathrm{~mA})$, they are commercially available and react in a short time. Due to the wide range of products and a lack of proper branding, we had to use a compilation of available datasheets for physically similar motors in order to estimate power consumption and operating voltage.

\section{DEVELOPMENT}

The first stage of development was to test the Freenect driver under Ubuntu Linux, this had to be done using a remote session via the Secure Shell (SSH) protocol due to the fact that the BeagleBone does not have a display output. Once installed and tested, we divided the screen in 6 imaginary sections (Figure 1) which will be mapped to an output vibrator.

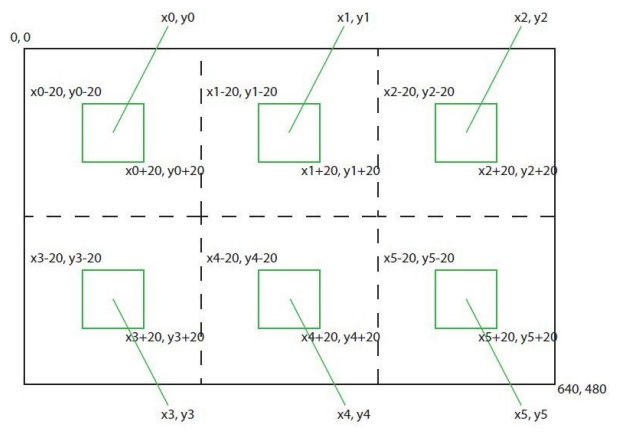

Figure 1: Imaginary screen divisions.

We calculate the minimum distance found in each screen section, given this distance value we can assign a frequency for the vibration pulses. When an object is far from the user, then the frequency of pulses will be small. As the object approaches, this frequency is to be increased; when the object is at a distance that is less than a meter, then all pulses stop, thus indicating an imminent threat. This behavior is illustrated in Figure 2.

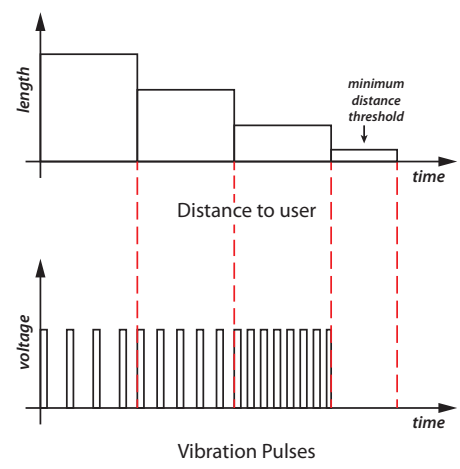

Figure 2: Top: object to device distances. Bottom: Corresponding vibration pulse frequency.
The proposed hypothesis is that, given enough training, a person may be able to substitute the lack of visual information with this pulses. The goal is not to make a virtual eye, but a very basic depth information sensor that can produce real-time outputs that the brain would then read as visually as possible.

Once this behavior was defined and implemented, we had to transform the outputs to vibrations. We initially tried to power the motors with whatever power the BeagleBone would deliver, but most of the motors did not emit a perceivable vibration. This lead to the design of a simple power circuit which will act as an electronically activated switch (Figure 3). This circuit proved to be sufficient to make the vibrators work and was replicated for every output.

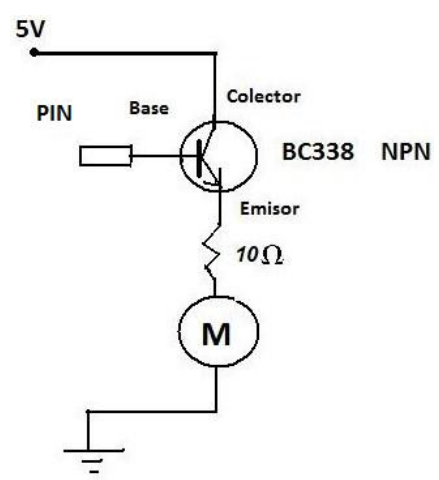

Figure 3: Motor driver circuit.

Information flows as follows (Figure 4), the Kinect samples distances and estimates them for a full frame $(640 \times 480$ pixels $)$. This frame is divided in six imaginary regions, from which six corresponding minimum distances are calculated. Each of this minimum distances is mapped to vibration pulses, whose frequency changes inversely depending on the measured distance. This process is repeated 30 times per second, as this is the native frame-rate the Kinect sensor uses. The fully assembled device is shown in Figure 5.

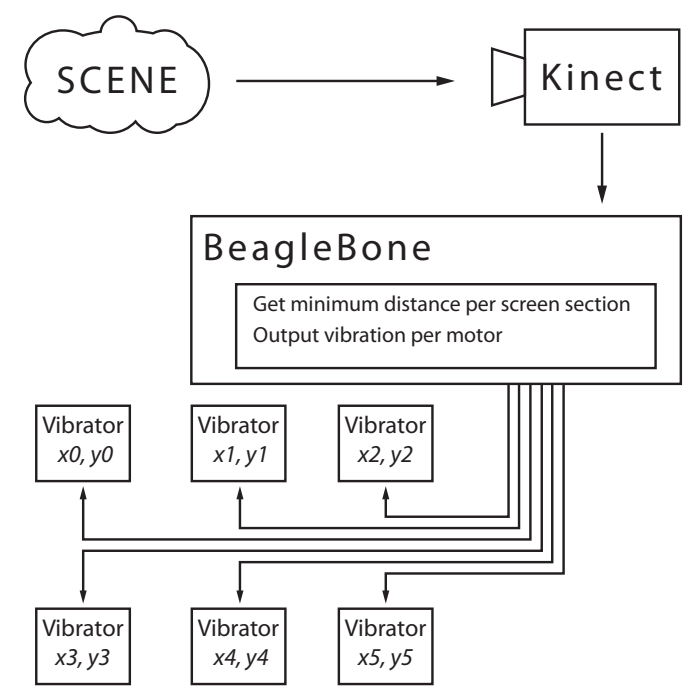

Figure 4: Information flow. 


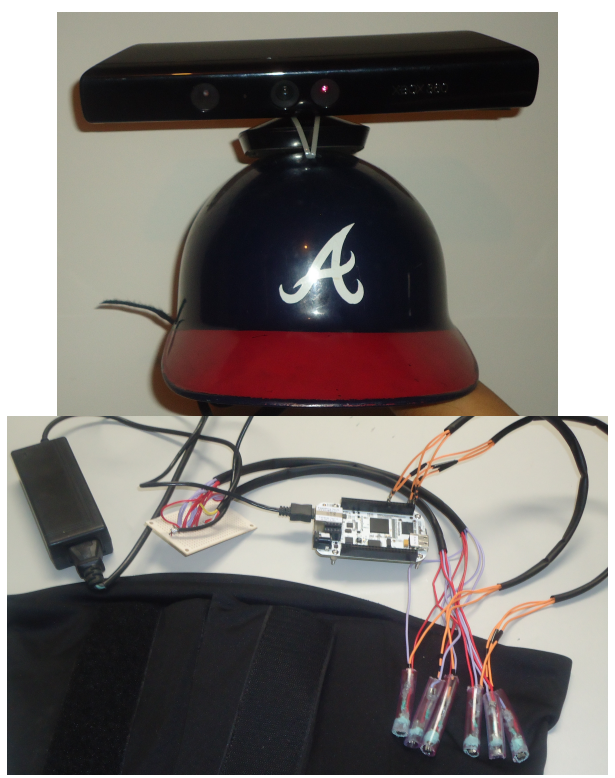

Figure 5: Fully assembled device.

\section{TEST CASES}

Initial tests where done by one of the researchers to verify the proper function of our device. During this trials we corroborated that the Kinect is an indoors-only device and when the apparatus is exposed to sunlight, distance estimation gets erratic and it returns a matrix full of the blank distance data for every pixel.

After this first tests, the device was given to people in different environments (a television studio, work offices and a computer laboratory). Figure 6 shows one of the participant test subjects.

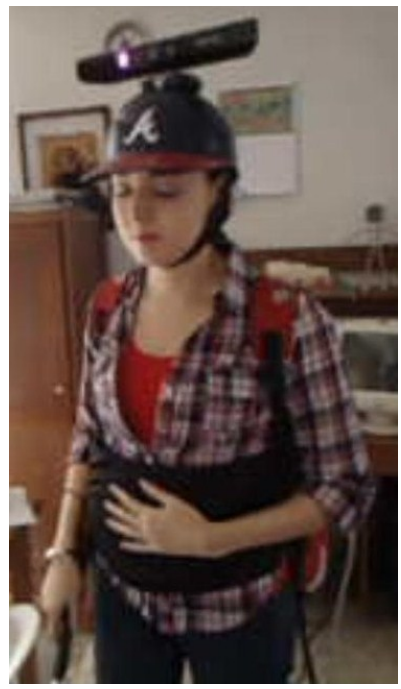

Figure 6: Test user

Some of the test users suggested that the placement of the Kinect sensor may not be optimal, though the proposed solution (putting it on the chest area) is probably not a good one. The idea behind placing the Kinect on the head is to allow the user to rapidly change its point of view, while putting it on the chest may hamper this ability.

Further tests exposed an unexpected weakness, we had placed the vibrators too close to each other for them to be sufficiently identifiable. This lead to alter the device and place the motors further apart from each other, two on each side of the torso and two on the abdominal region.

It is important to notice that no training was given to test users. This indicated that the device could be used instantly, though with proper experience, a visually impaired person may have no problems at all to use it [6]. At the time of writing this paper, we require further testing with a more diverse audience and in completely unknown scenarios. The later because we suspect that users memory may have hampered their judgement on the effectiveness of our device.

\section{CONCLUSIONS AND FUTURE WORK}

\section{A. Conclusions}

Commercially available hardware is good enough to create several projects, including open hardware prosthesis. Our device is based on the Kinect sensor, which can only work on indoor environments and is a closed source device, though is cheap enough to experiment with. The BeagleBone is expensive compared to other barebones, but it has built-in GPIOs giving it the advantage of having everything we needed to process and output information in a single board.

With this research project we combined computer vision and electronics to create a prosthetic device that is economically feasible and, if one was to massively manufacture, this costs would go even further down.

The human brain's plasticity [6] is a key component for our device to work, recent studies have found that neural pathways are rerouted, leading to the "replacement" of, say, sight via sound or sight via tongue nervous terminals (taste). Our device works under the assumption that, with sufficient training one could replace (to an extent) one sense with another.

Our goals where to create a device that could be built with commercial hardware for a low price. That this device could transform depth measurements into vibrating outputs that a visually impaired person could interpret. This goals where fully accomplished.

We wanted to create an apparatus that worked both indoors and outdoors, this was not a hard goal in the sense that solving the problem for either condition was sufficiently good. We got good results for indoor environments.

\section{B. Future work}

Replacing the input for our sensor is our number one priority, not because it has poor performance, but because it does not work properly outdoors. We have been looking at some other options for 3D sensing [7], but they seem to require additional computational power. Another option for this stage of the device's information flow is to use ultra-sound based sensors, but they're prone to error.

Moving from a solely ARM based barebone to a more complex one, such as NVidia's Tegra platform which includes 
parallel processors in the form of Graphics Processing Units (GPU), would allow our device to run more demanding algorithms for depth estimation.

\section{REFERENCES}

[1] BeagleBone Community. Beaglebone system reference manual rev a6. [Online]. Available: http://beagleboard.org/bone?

[2] Microsoft Robotics. Kinect sensor specifications. [Online]. Available: http://msdn.microsoft.com/en-us/library/hh438998.aspx

[3] R. Manduchi and J. Coughlan, "(computer) vision without sight," Commun. ACM, vol. 55, no. 1, pp. 96-104, Jan. 2012. [Online]. Available: http://doi.acm.org/10.1145/2063176.2063200

[4] Arduino. Arduino web page. [Online]. Available: http://arduino.cc

[5] O. Community. Openkinect project. [Online]. Available: http:// openkinect.org/wiki/Main\%20Page

[6] A. Amedi, N. Raz, P. Pianka, R. Malach, and E. Zohary, "Early 'visual' cortex activation correlates with superior verbal memory performance in the blind," Nature Neuroscience, vol. 6, pp. 758-766, 2003.

[7] Y. Y. Schechner and N. Kiryati, "Depth from defocus vs. stereo: How different really are they?" Int. J. Comput. Vision, vol. 39, no. 2, pp. 141-162, Sep. 2000. [Online]. Available: http://dx.doi.org/10.1023/A:1008175127327

[8] K. Khoshelham, "Accuracy analysis of kinect depth data." [Online]. Available: https://wiki.rit.edu/download/attachments/52806003/ 1s2011\%20submission\%2040.pdf

[9] D. S. Ladislao Mathe and G. Gomez. Estudio del funcionamiento del sensor kinect y aplicaciones para bioingeniería. [Online]. Available: http://www.computacion.efn.uncor.edu/sites/default/files/123.pdf

[10] A. Nagai. How to program a beaglebone on $\mathrm{c} / \mathrm{c}++$. [Online]. Available: http://www.cs.gunma-u.ac.jp/\%7enagai/wiki/index. php?BeagleBone $\% 20 \%$ A4\%CE\%BB\%C8\%A4\%A4\%CA\%FD

[11] J. J. Castellanos López. Installing libfreenect under Linux. [Online]. Available: http://www.youtube.com/watch?v=LgY2zf5qKnA

[12] D. Molloy. Beaglebone: Gpio programming on arm embedded linux. [Online]. Available: http://www.youtube.com/watch?v=SaIpz001E84

[13] M. Richardson. How-to: Get started with the beaglebone. [Online]. Available: http://www.youtube.com/watch?v=Y0uqRVxismQ

José Ortiz Bejar got a B.Sc. degree in Electrical Engineering from the Universidad Michoacana de San Nicols de Hidalgo in 2005. In 2008 he got a M.Sc. degree in Electrical science from the UMSNH. His research includes Web development, Operative systems administration and Artificial Intelligence.

Garibaldi Pineda Garca got a B.Sc. degree in Electrical Engineering from the Universidad Michoacana de San Nicols de Hidalgo in 2005. In 2008 he got a M.Sc. degree in Electrical science from the UMSNH. Some of his research deals with human-computer interaction, software for mobile devices and web development.

José Francisco Mercado Miramontes got a B.Sc. degree in Computer Engineering from the Universidad Michoacana de San Nicols de Hidalgo in 2013. His research interests are networking and mobile devices. 